\title{
La contratación temporal de trabajadores altamente calificados H-1B en Estados Unidos: surgimiento, evolución y condiciones actuales
} Temporary Hiring of Highly Qualified H-1B Workers in the United States: Emergence, Evolution, and Current Conditions

Paz Trigueros Legarreta*

\section{RESUMEN}

La finalidad de este artículo es estudiar el programa de visas de trabajo temporal H-1B, la forma en la que surgió y sus transformaciones a través del tiempo, así como su diversidad y características actuales; los ataques de distintos sectores de la población, además de la contradictoria política del presidente Trump en relación con la contratación de trabajadores extranjeros calificados, y principalmente de la que se refiere a las visas H-1B, que han sido las más criticadas desde su creación en 1990.

Palabras clave: visas de trabajo temporal, migración calificada, cambios tecnológicos, política migratoria, visas $\mathrm{H}-1 \mathrm{~B}$.

\section{ABSTRACT}

This article examines the H-1B temporary work visa program, how it emerged, and its changes over time; its diversity and current characteristics; the attacks from different sectors of the population, as well as President Trump's contradictory policy regarding the hiring of qualified foreign workers and, mainly the $\mathrm{H}-1 \mathrm{~B}$ visas, which have been the most criticized since their creation in 1990.

Key words: temporary work visas, highly skilled migration, technological changes, immigration policy, $\mathrm{H}-1 \mathrm{~B}$ visas.

\footnotetext{
* Profesora-investigadora, Departamento de Sociología, Universidad Autónoma Metropolitana, unidad Azcapotzalco (UAM-A), <ptl@correo.azc.uam.mx>.
} 


\section{INTRODUCCIÓN}

Como ya ha sido mencionado en varios artículos de este número, la importación de trabajadores altamente calificados se ha incrementado notablemente en los últimos tiempos en la mayoría de los países desarrollados. Sin embargo, también en estos años se ha recurrido cada vez más a su utilización de manera temporal, durante sus años de mayor productividad, y sólo en algunos casos se les otorga la residencia definitiva.

Estados Unidos no es la excepción, y aunque sólo se implementó esta forma de contratación en la segunda mitad del siglo xx, es a partir de 1990 cuando sus números crecieron de manera sorprendente. Es justo ese año cuando se incorporan cambios relevantes, no sólo en términos cuantitativos, sino en relación con el tipo de trabajadores que se importan, la forma de contratación, las exigencias y las modalidades en las que pueden acceder a la residencia definitiva (green card).

Los cuestionamientos a este tipo de contrataciones siempre han estado presentes, pero con el advenimiento del presidente Trump se incrementaron, así como a los nuevos ordenamientos para modificarlos, como parte de su política de "America first".

En este artículo pretendo realizar un seguimiento de la forma en que surgen estas visas para trabajadores calificados de manera temporal en Estados Unidos, ubicándolas en el contexto en el que aparecen, así como sobre sus principales transformaciones a través del tiempo, para terminar con los cuestionamientos más recientes y las modificaciones que se han instaurado desde la llegada de Trump a la presidencia.

\section{LOS ANTECEDENTES DE LA CONTRATACIÓN DE TRABAJO CALIFICADO TEMPORAL EN Estados UNIDOS}

Debido a la amplitud de su territorio, Estados Unidos se caracterizó siempre por la atracción de extranjeros, principalmente europeos, para poblar el país y satisfacer los requerimentos laborales de su naciente industria. A la mayoría se les recibía como residentes y en pocos años se les otorgaba la ciudadanía. Sin embargo, existieron formas de importación mucho menos amistosas, como es el caso de los trabajadores orientales en California en las últimas décadas del siglo XIX, y que finalmente fueron expulsados; de los inmigrantes mexicanos que eran recibidos de manera temporal para realizar trabajo agrícola o en la instalación de las vías férreas en la parte occidental del país, a los que nunca se les dio ningún documento de residencia y, sobre todo, de los africanos, que fueron llevados violentamente como esclavos. En general, había mucho rechazo a la contratación de trabajadores temporales, sobre todo por 
parte de las organizaciones sindicales estadunidenses, por lo que ésta fue prohibida desde 1885 mediamte la Alien Contract Labor Law (Jachimowicz y Meyers, 2002; Wong, 2010; Trigueros, 2015). Era usual que se admitieran extranjeros altamente calificados que huían de su país debido a las guerras, la persecución política o simplemente por motivos económicos. Se les recibía de manera definitiva y se les facilitaba su integración en universidades, centros de investigación y demás fuentes de trabajo.

Sin embargo, las cosas comenzaron a cambiar con la segunda guerra mundial y, sobre todo, a partir de la aprobación de la McCarran-Walter Act de 1952, ${ }^{1}$ que por primera vez incluyó la contratación de trabajadores temporales. Se crearon para ello las visas " $\mathrm{H}$ ", que se dividieron en $\mathrm{H}-1$ para trabajadores especializados y $\mathrm{H}-2$ para aquellos que contaban con escasa calificación. En ese entonces no era necesario demostrar que no había trabajadores en Estados Unidos que pudieran ser perjudicados con la contratación de extranjeros, ni se estableció ningún tope en su número. Lo único que se exigía era que tanto el trabajo como el trabajador que ocupara ese puesto fueran temporales (Meyers, 2006: 4) $)^{2}$.

De acuerdo con el Servicio de Inmigración y Naturalización (Immigration and Naturalization Service, INS), se consideraban como "trabajadores especializados" a aquellos extranjeros con cualidades y capacidades notables (aliens of "distinguished merit and ability") que iban a Estados Unidos a realizar servicios de una naturaleza excepcional. La lista de actividades que incluía era muy variada. El Anuario del ins de 1978 registró 6838 inmigrantes aceptados en esta categoría, entre los que destacaban los músicos y compositores (el 20.4 por ciento) y las enfermeras registradas (16.4), seguidos por los atletas y actividades asociadas (5.7 por ciento), los ingenieros (2), los bailarines (2) y muchos más (USDOJ, 1980).

También llegaban trabajadores calificados temporales con otras visas de "no inmigrantes", como "extranjeros de intercambio" o "transferidos intracompañías". 3

El número de visas H-1 emitidas fue aumentando en la década de los ochenta, de tal manera que, según los anuarios estadísticos del INS, pasaron de 16838 en 1978 a 114467 en 1991, último año en que funcionaron con la clasdificación H-1, o sea, la de "aliens of distinguished merit and ability". A partir de 1992 entraron en vigor las visas $\mathrm{H}-1 \mathrm{~B}$, a las que se definieron de una manera más precisa, como veremos más adelante, debido a las quejas sobre la forma en que se otorgaban, y que se hicieron más

\footnotetext{
${ }^{1}$ Esta ley también es importante porque estableció los dos pilares en los que se basa el sistema migratorio estadunidense: la reunificación familiar y la inmigración basada en el empleo.

${ }^{2}$ Esta exigencia fue suprimida por el Congreso en una ley de abril de 1970, con la finalidad de ampliar el abanico de opciones de contratación (Jachimowicz y Meyers, 2002; Wasem y Collver, 2001: 2 y 5; y Wasem, 2006, 2).

${ }^{3}$ Esta categoría fue creada en la Ley del 3 de abril de 1970 (Jachimowicz y Meyers, 2002; Wasem y Collver, 2001: 2, 5; Wasem, 2006: 2).
} 
especializadas en la medida en que avanzaban los cambios científicos y tecnológicos en los centros de investigación, universidades y empresas enfocadas a lo que, desde entonces, se conoce como sTEM (ciencia, tecnología, ingeniería y matemáticas -science, technology, engineering, mathematics-). En una publicación anterior (Trigueros, 2012b) hago referencia a la que se ha llamado revolución científica y tecnológica (RCT), que ha dado lugar a la aplicación de nuevos conocimientos a los sistemas productivos; ${ }^{4}$ en este sentido, la mencionada RCT se distingue sobre todo por el hecho de que la ciencia precede a la tecnología, y ésta a la producción, razón por la cual la actividad científica se constituye como el prerrequisito directo para el avance de las fuerzas productivas, convirtiendo en obsoletas a las técnicas anteriores (Corona, 1991).

\begin{tabular}{|lcc|}
\hline \multicolumn{3}{|c|}{ Cuadro 1} \\
TRABAJADORES EXTRANJEROS CON CUALIDADES Y CAPACIDADES NOTABLES, 1978 \\
& H-1 & $\begin{array}{c}\text { Trabajadores extranjeros } \\
\text { con cualidades y capacidades } \\
\text { notables (\%) }\end{array}$ \\
Total & 16838 & 100.0 \\
Músicos y compositores & 3431 & 20.4 \\
Enfermeras acreditadas & 2744 & 16.3 \\
Atletas y actividades asociadas & 955 & 5.7 \\
Bailarines & 406 & 2.4 \\
Ingenieros & 335 & 2.0 \\
Ciencias de la vida y físicas & 254 & 1.5 \\
Médicos, dentistas y trabajadores relacionados & 197 & 1.2 \\
Profesores de universidades y de colleges & 193 & 1.0 \\
Pintores y escultores & 162 & 0.9 \\
Profesores no universitarios & 157 & 0.9 \\
Investigadores & 145 & 0.2 \\
Especialistas en cómputo & 30 & 46.5 \\
Otros* & 7829 & \\
* Otros incluye una gran diversidad de actividades, que van desde profesionistas y administradores hasta em- \\
pleados, artesanos y pasteleros, aunque todos en muy pequeñas cantidades. \\
Fuente: usdos (1986). & & \\
& & \\
\end{tabular}

\footnotetext{
${ }^{4}$ Según Corona, esta revolución incluye cuatro procesos diferentes: la cibernetización, que se refiere a la retroalimentación de la información mediante centros de control por computadora; la quimización, por la cual la materia prima es transformada de manera continua; la biotecnología, que se enfoca en la utilización de microorganismos para la transformación de las materias, y la aplicación de los procesos energéticos (Corona, 1991: 18-21).
} 
Ante este panorama, se imponía un cambio en la legislación migratoria que incorporara las nuevas exigencias, para que la Unión Americana no quedara atrás de otros países que ya estaban adaptando sus regulaciones en la materia en ese sentido. Aunque ya en 1989 se habían modificado las visas H-1, ${ }^{5}$ fue con la Immigration Act de 1990 que el Congreso respondió de una manera integral a las nuevas exigencias.

\section{LA IMPORTANCIA DE LA LEY DE INMIGRACIÓN DE 1990 (IMMACT 1990)}

A diferencia de la ley llamada IRCA (Immigration Reform and Control Act) de 1986, cuya finalidad era resolver el problema de la migración indocumentada, principalmente de personas con baja calificación, la ley de 1990 buscaba un nuevo esquema migratorio que modificara las bases del sistema establecido en 1952, que daba prioridad a la reunificación familiar. Con ella se intentaba brindar mayor atención al otro pilar del sistema migratorio: la migración laboral, y así responder a las exigencias de los distintos tipos de empleadores, con la finalidad de evitar que el país también quedara rezagado en esa materia. Es por ello que se incrementó la proporción de inmigrantes que podían ser aceptados bajo la modalidad de requerimientos laborales, que pasó del 10 por ciento del total anual, al 21 por ciento (lo que significaba aumentar de cincuenta y cuatro mil a ciento cuarenta mil personas anualmente) (Alarcón, 2000: 2).

Se estableció un sistema de preferencias de contratación en el que la primera se destinaba a trabajadores con habilidades extraordinarias en distintas ramas del conocimiento, el arte y el deporte (cuarenta mil); la segunda preferencia, a personas con grados avanzados o habilidades excepcionales en las ciencias, artes o negocios (cuarenta mil), y la tercera a profesionistas con, cuando menos, el título de licenciatura, y a trabajadores calificados (treinta mil), lo que sumaba ciento diez mil green cards para personal altamente calificado (Alarcón, 2000: 4; Mehta, 2004; Trigueros, 2012b).

Sin embargo, estas visas eran insuficientes para atender las necesidades del capital, por lo que se recurrió al uso intensivo de permisos de trabajo temporal, que habían sido tan rechazados desde principios del siglo xx puesto que iban en contra de la filosofía de inmigración tradicional. Algunas de estas visas fueron creadas para este fin y otras, ya existentes, simplemente fueron modificadas. Con ellas se buscaba probar a los trabajadores antes de darles la residencia definitiva y seleccionar

\footnotetext{
${ }^{5}$ El principal cambio fue la creación de la categoría H-1A, a la que se puso el nombre nursing relief, para atender la creciente escasez de enfermeras altamente preparadas, mientras que los demás migrantes especializados entrarían como H-1B (Meyers, 2006: 4).
} 
únicamente a los que se adaptaran mejor a los requerimientos del país y de los empresarios. Por este motivo, se estableció el llamado "intento dual", que se refería al derecho que tienen estos trabajadores de solicitar simultáneamente una visa permanente, por lo que no se les exigía, como en otras visas temporales, demostrar que cuentan con residencia en su país de origen.

\begin{tabular}{|ccc|}
\hline \multicolumn{4}{|c|}{ Cuadro 2 } \\
SISTEMA DE PREFERENCIAS PARA ASIGNAR VISAS \\
A INMIGRANTES CALIFICADOS
\end{tabular}

Las visas que han atraído más la atención de empleadores, gobierno y demás interesados en el tema son las $\mathrm{H}-1 \mathrm{~B}$ para científicos y técnicos especializados. Para que cumplieran con las nuevas expectativas, se definieron mejor sus requerimientos y se ampliaron las exigencias de contratación, al especificar que sólo se asignarían a trabajadores que desempeñaran las consideradas "ocupaciones especializadas", lo que significaba la aplicación teórica y práctica de un cuerpo de conocimientos especializados, por lo que usualmente se requería, cuando menos, de un título de licenciatura (o su equivalente). También se exigía una licencia para trabajar en Estados Unidos cuando fuera requerida en su campo de especialización (por ejemplo, arquitectos o terapeutas físicos), o bien tener, según determinara la autoridad competente, el equivalente al grado requerido, a través de una combinación de educación, entrenamiento y/o experiencia. Se incluyeron más de cuarenta profesiones, ${ }^{6}$ aun cuando,

\footnotetext{
${ }^{6}$ Entre ellas están los ingenieros, contadores públicos, médicos, profesores universitarios, científicos, arquitectos, abogados, enfermeras, técnicos de laboratorio, y técnicos médicos o clínicos; así como los modelos, para quienes se exige tener "mérito distinguido o habilidad excepcional".
} 
en la práctica, una amplia mayoría se asignan a analistas y programadores de sistemas computacionales (DHS, 2003: 94).

El cuadro 3 nos muestra las principales visas de trabajo temporal, entre las cuales sólo las H-2A y H-2B son para trabajadores no calificados. Las visas O-1 y O-2 se asignan a "trabajadores con habilidades extraordinarias y logros importantes", que se distinguen en ciencias, artes, educación, negocios o deportes; así como para productores cinematográficos o de televisión y sus acompañantes y asistentes; las visas $\mathrm{P}$, para atletas, entrenadores y artistas reconocidos internacionalmente, o dentro de algún programa de intercambio o "culturalmente único"; las visas $Q$, para participantes en programas internacionales de intercambio cultural, y las $\mathrm{R}$, para realizar trabajos de carácter religioso (DHS, 2003: 120, nota 9; Trigueros, 2012b: 161).

\begin{tabular}{|llrr|}
\hline \multicolumn{5}{|c|}{ Cuadro 3 } \\
ADMISIONES DE TRABAJADORES TEMPORALES Y EN ENTRENAMIENTO, \\
Visa & \multicolumn{1}{|c}{ SEGÚN EL TIPO DE VISA (2016) } \\
Categorías de trabajadores & Absolutos & \multicolumn{1}{c|}{$\%$} \\
H-1B & Trabajadores en ocupaciones "especiales" & 534633 & 19.29 \\
H-2A & Trabajadores agrícolas & 348052 & 12.56 \\
H-2B & Trabajadores no agrícolas & 119457 & 4.31 \\
H-3 & Trabajadores en entrenamiento industrial & 2786 & 0.10 \\
O-1 y O-2 & Trabajadores con habilidades/logros extraordinarios & 130589 & 4.71 \\
P1, P2 y P3 & y asistentes & 125406 & 4.52 \\
Q-1 & Trabajas y artistas & 3038 & 0.11 \\
R-1 & Trabajadores en programas de intercambio cultural & 14095 & 0.51 \\
TC y TN & Trabajadores profesionales, acuerdos Canadá y TLCAN & 816149 & 29.44 \\
L-1 & Transferidos intracompañías & 677583 & 24.45 \\
Total & & 2771788 & 100.00 \\
Fuente: Elaboración propia con datos del Yearbook of Immigration Statistics 2016, tabla 25 (DHs, 2017a). \\
\hline
\end{tabular}

Es necesario mencionar que actualmente se consideran las visas L-1 como visas de trabajo porque se ha vuelto usual contratar con ellas a expertos en computación y sistemas para enviarlos a dar servicio a los clientes de las empresas, sin tener que pasar por los complicados trámites de las visas H-1B. Fueron creadas en 1970 para facilitar la transferencia de personal del nivel más alto en las compañías multinacionales y ocuparlo en el funcionamiento de la firma a nivel global. En la ley de 1990 se 
efectuaron algunas modificaciones para evitar los abusos que ya existían entonces. Se precisó que "conocimiento especializado" se refería al producto de la empresa; se fijaron hasta siete años como límite para la estancia de gerentes y ejecutivos y cinco para aquellos que eran aceptados por tener conocimientos especializados acerca del producto (Wasem, 2006: 2).

De cualquier manera, los abusos continúan a pesar de las quejas de investigadores y de trabajadores estadunidenses, lo que no ha impedido que actualmente su número sea mayor que el de los trabajadores $\mathrm{H}-1 \mathrm{~B}$.

Entre los cambios motivados por la globalización destacan las visas que han surgido de los convenios y tratados internacionales realizados por la Unión Americana con otros países. La más importante, la visa TN, surgió como parte de la firma del Tratado de Libre Comercio de América del Norte (TLCAN), entre Canadá, Estados Unidos y México, que entró en vigor el 1 de enero de 1994. Según el Departamento de Seguridad Interna (DHS), con ella se facilita la entrada temporal de ciudadanos mexicanos y canadienses para actividades de negocios, intercambio comercial, así como para inversionistas y transferidos intracompañías (DHS, 2003: 94). Sin embargo, en este caso sus portadores no pueden optar por la residencia definitiva.

Por otro lado, las condiciones que ofrecen a cada uno de los socios han sido muy desiguales. Mientras que los mexicanos deben realizar complicados trámites en su país para que puedan acceder a esas visas, los canadienses las pueden obtener al cruzar la frontera, por lo que su número es mucho mayor. Esta facilidad ha dado lugar a que sea muy difícil cuantificar cuántos ciudadanos de Canadá las reciben, puesto que sólo se conoce su número de entradas, las cuales pueden ascender a varias en un año. En cambio, el Departamento de Estado no puede publicar cuántas visas se asignaron a estos solicitantes dado que únicamente registra las que otorgan sus representaciones diplomáticas en los lugares de origen.

\section{REQUERIMIENTOS Y EVOLUCIÓN DE LAS VISAS TEMPORALES DE TRABAJADORES CALIFICADOS Y CARACTERÍSTICAS ACTUALES}

Como ya se mencionó, la IMMACT de 1990 estableció muchas exigencias para aprobar las visas de trabajo temporal en general, pero entre las calificadas, las H-1B fueron las más reglamentadas y las que debían pasar por más filtros antes de que el trabajador seleccionado la tuviera en su poder. ${ }^{7}$

\footnotetext{
${ }^{7}$ Las visas H-2, que son las únicas orientadas a atraer trabajadores no calificados, también han sido muy reglamentadas, sobre todo las H-2A, creadas con la IRCA de 1986, que se destinan a la contratación temporal de trabajadores agrícolas (Trigueros, 2015).
} 
Los empleadores que las requieren tienen que cumplir un complicado proceso que incluye: presentar una solicitud al Departamento de Trabajo (Department of Labor, DOL) en la que se especifique el trabajo, salario, duración y lugar del empleo; para asegurar la protección de los trabajadores estadunidenses, atestiguar que pagarán más del salario prevaleciente o real y que están dispuestos a proporcionar las mismas condiciones que rigen para los trabajadores locales; garantizar que no exista ninguna huelga o cierre patronal, además de colocar un aviso sobre el empleo para asegurar que no existan trabajadores locales interesados en ese puesto (Mehta, 2004: 31).

Después de recibir el certificado, el empleador debe llenar una solicitud ante el Departamento de Seguridad Interna (Department of Homeland Security, DHS) para patrocinar a un extranjero, ya sea que se encuentre fuera de Estados Unidos o dentro del país, laborando para otro empleador o realizando otra ocupación. Su contratación puede ser de hasta tres años inicialmente, con posibilidad de una extensión que no exceda otros tres años (DHS, 2003: 95). El siguiente paso queda en manos del candidato a obtener la visa, quien debe solicitarla al Departamento de Estado (Department of State, DOs) a través de alguno de sus consulados. Para ser aceptado, el solicitante tiene que entrevistarse con un cónsul en su país de residencia, quien verifica que la documentación requerida sea consistente. Si reúne las condiciones, se le otorga la visa. En el caso de los que solicitan una ampliación o modificación de su visa inicial, pueden hacer el trámite sin salir del país (Ruiz et al., 2012: 4-5).

Los solicitantes que entran con estas visas tienen condiciones mucho mejores que las de los trabajadores no calificados. Se les brinda la posibilidad de cambiar de empleo cuando el patrón no cumple con lo ofrecido o simplemente encuentren un trabajo mejor; tienen libertad de movimiento en el país y de salir y entrar cuantas veces quieran, así como la facilidad para llevar a sus familiares, aunque no para que trabajen, a menos que consigan otra visa que se los permita. Lo que es más importante, se les abre la posibilidad de que, al final del periodo de contratación -que puede extenderse hasta por seis años- soliciten una visa permanente con el patrocinio de su empleador (doble intento) (Trigueros, 2012a: 159). Sin embargo, no siempre se cumple con las condiciones ofrecidas y, en lo que se refiere al otorgamiento de la residencia definitiva, en la realidad existe un gran retraso (backlog) debido a los límites impuestos por la legislación. Mientras un trabajador consigue su green card debe permanecer con su patrón en calidad de trabajador $\mathrm{H}-1 \mathrm{~B},{ }^{8}$ pues es el único que puede

\footnotetext{
${ }^{8}$ Preston (2015) cita los ejemplos de un ingeniero eléctrico proveniente de India que llevaba trece años esperando su green card, y del doctor Sengupta, que era profesor de medicina en la Universidad de Boston y tenía un equipo de investigación avanzada que atraía pacientes de todo el mundo, pero que, después de diez años de solicitar la visa definitiva sin conseguirlo, en 2015 le negaron la renovación de su visa H-1B, por lo que en dos meses debía abandonar el país.
} 
apoyarlo (Ruiz et al., 2012: 3). El presidente Obama trató de solucionar el problema a través de sus órdenes ejecutivas, solicitando al Dos que acelerara el proceso; sin embargo, los funcionarios se vieron impedidos debido a que las solicitudes sobrepasaban con mucho la cantidad de ciento cuarenta mil que permite la ley distribuir anualmente. Además, también está establecido que ningún país reciba más del 7 por ciento de estas visas, por lo que los inmigrantes de India y China deben esperar hasta por una década, o más (Preston, 2015). Es por eso que dice Philip Martin (2003) que la puerta de las visas temporales está muy abierta, pero la de las visas de inmigrantes es mucho más angosta, por lo que el portador de una H-1B puede quedar sometido a una relación de servidumbre durante varios años.

Por otro lado, con el transcurso de los años las quejas contra estas visas han proliferado por parte de diversos actores sociales. Entre ellos destacan los sindicatos y las asociaciones profesionales, que demandan un mayor control de las solicitudes y del tipo de extranjeros que llegan con ellas, así como la exigencia de que se generen programas para la formación de especialistas capaces de realizar los trabajos que, se dice, no existen en Estados Unidos. Martin (2003) señala que desde la crisis de la industria de la tecnología en 2000, muchos empresarios reemplazan legalmente a trabajadores estadunidenses con trabajadores $\mathrm{H}-1 \mathrm{~B}$ y hasta se dan casos en los que han sido forzados a entrenar a portadores de dichos visados antes de despedirlos, como lo hizo la empresa Siemmens. Por su parte, Hira (2011) menciona que siete de las diez principales firmas empleadoras de inmigrantes H-1B de los años 2007-2009 realizaban outsourcing con ellos. ${ }^{9}$ También existen quejas por parte de los empleadores, por lo complicado de los trámites, pero sobre todo porque consideran que el número de contrataciones autorizado es insuficiente, ya que la ley estableció un límite anual de sesenta y cinco mil, para proteger a los trabajadores locales.

En respuesta, han surgido diversas leyes que tratan de atender a los quejosos y subsanar, o al menos paliar, los efectos negativos en su asignación y en su número. En 1998 la American Competitiveness and Workforce Improvement Act (ACWIA) aumentó el tope a 115000 para los años 1999 y 2000, y a 107500 en 2001.

A cambio de estas ampliaciones, se impuso una cuota de quinientos dólares por cada trabajador que solicitaran, con la finalidad de obtener dinero para apoyar los programas de educación y entrenamiento que facilitaran el aumento de la oferta local a trabajadores en STEM.

Con la Ley Pública 106-311 (del 17 de octubre de 2000), se aumentó esta cuota a 1000 dólares por cada trabajador, y se extendió su aplicabilidad a las peticiones realizadas hasta el 30 de septiembre de 2003, además de que se incrementó el número de

\footnotetext{
${ }^{9}$ Las siete compañías que realizaban outsourcing eran Infosys, Wipro, Satyam, Tata, Cognizant, IBM y Accenture, en tanto que las tres que no lo hacían eran Microsoft, Deloitte e Intel (Hira, 2011).
} 
solicitudes a 195000 para 2001, 2002 y 2003, aunque se señalaba que después de esa fecha volverían al tope inicial de 65000. Asimismo, se exentó de estos topes a las organizaciones de investigación no mercantiles o gubernamentales; a las instituciones de educación primaria, secundaria y superior y otras entidades educativas no lucrativas, así como a los empleadores que solicitaran la extensión o modificación de la estancia de algún extranjero, o para corregir un error (Meyers, 2006: 5).

En 2004 se produjeron nuevos cambios: la H-1B Visa Reform Act de ese año instituyó la que llamaron "Cuota para la prevención y detección de fraudes" (fraud fee), de quinientos dólares, que debían pagar los solicitantes junto con la petición inicial, tanto de visas H-1B como de L-1, que han sido tan cuestionadas, aunque no aplicaba a las solicitudes de extensión o modificación de la estancia de estas categorías de trabajadores (Meyers, 2006). El 8 de diciembre se reinstituyó de manera permanente la cuota establecida en la ACWIA de 1998, que había terminado a fines de 2003, y se elevó a mil quinientos dólares por cada petición calificada. No alteró las exenciones y bajó la cuota a setecientos cincuenta a los empleadores que tuvieran no más de veinticinco empleados en Estados Unidos. Además, se hizo una nueva adición de veinte mil solicitudes, exentas del tope, cuando los solicitantes hubieran obtenido un grado de maestría o mayor en Estados Unidos (Meyers, 2006).

Otra medida que se tomó fue la instauración de la premium processing fee en 2006, con la cual el US Citizenship and Immigration Services (USCIS) garantizaba que en quince días calendario se procesaría la solicitud de extensión de la estadía para ciertas visas $\mathrm{H}-1 \mathrm{~B}$, para los empleadores que requerían de un proceso expedito, y que en 2017 era de 1225 dólares $^{10}$ (USCIS, 2017a; Ruiz et al., 2012). A pesar de todo, estos cobros no lograron inhibir el número de solicitudes. En el año fiscal de 2016 éstas llegaron a 399 000, lo que significó un aumento de 14 por ciento en relación con el año anterior. De ellas, se aprobaron 348 000, que representan un incremento del 25 por ciento respecto del periodo anual previo, de las cuales 114503 eran solicitudes iniciales y 230759 renovaciones ${ }^{11}$ (DHS, 2017b).

Es preciso mencionar que, aunque se hace hincapié en que deben ser trabajadores con alta calificación, de los aprobados en 2016, el 44 por ciento contaban únicamente con licenciatura, el 45 con maestría y tan sólo un 7 por ciento con doctorado (DHS, 2017b). Como se mencionó y ha sido una de las principales críticas de estas visas, la gran mayoría se asignó a trabajadores en análisis de sistemas, programación y otras actividades relacionadas con el cómputo (el 69.1 por ciento) (DHS, 2017b).

\footnotetext{
${ }^{10}$ Si no se cumplía con el tiempo, la agencia regresaría el pago al peticionario y continuaría con el proceso expedito de la aplicación.

${ }^{11}$ Sin embargo, como veremos más adelante, las medidas tomadas durante el primer año de la presidencia de Trump sí lograron bajarlas a 199000.
} 
En cuanto a los países de origen, como ya es de todos conocido India sobresale con la gran mayoría, que en 2016 ascendió a 256226 individuos, que constituyen el 74.2 por ciento del total; seguida muy de lejos por China con el 9.3. En niveles todavía más bajos se encuentran otras naciones asiáticas, como Corea del Sur (el 1 por ciento), Filipinas (el 1 por ciento) y Taiwán (un 0.6 por ciento). En cuanto a los Estados no asiáticos que participan, sus números son también muy reducidos: el Reino Unido con el 1.1 por ciento; Canadá con el 1 por ciento y México con un 0.6 por ciento (DHS, 2017b).

Para conocer las principales empresas que demandan este tipo de visas, utilizamos la información de la oficina de Administración de Empleo y Capacitación (Employment and Training Administration -ETA-), perteneciente al DOL, de 2016. De las tres que ocupaban los primeros lugares, las dos primeras solicitaban analistas de sistemas computacionales. En primer lugar, estaba Deloitte Consulting LLP, la cual obtuvo la certificación de 164000 posiciones, número muy superior a las siguientes empresas certificadas: Cognizant Technology Solutions U.S. Corporation, con 97 472, y PricewaterhouseCoopers LLP, que obtuvo 54093 certificaciones para contadores y auditores (OFLC, 2016: 31).

Sin embargo, junto con estas grandes empresas existe un gran número de establecimientos que requieren de sólo unas pocas posiciones, incluso muchos de ellos nada más una. Sobresalen las enfocadas en el sector educativo, ${ }^{12}$ que aunque constituyen únicamente el 2.5 por ciento de todos los puestos requeridos, son muy importantes para las instituciones de educación de todos los niveles. De éstas, aproximadamente el 75 por ciento fueron solicitadas por universidades y colegios profesionales; en tanto que cerca de 18 por ciento fueron para escuelas elementales y secundarias (OFLC, 2016: 37).

Aunque se puede considerar que están muy bien pagadas las actividades que realizan los trabajadores especializados en cómputo, la mediana de su remuneración anual en 2016 ascendió ochenta y dos mil dólares, por lo que no son, de ninguna manera, las mejor retribuidas. Los superan los abogados (cuya mediana de ingresos anuales es de ciento quince mil dólares); los ocupados en las "ciencias de la vida" (ciento seis mil); en las ciencias sociales (cien mil); los arquitectos, ingenieros y topógrafos (ochenta y siete mil), y los contratados en el campo de medicina y salud (ochenta y seis mil) (DHS, 2017b: 17).

\footnotetext{
${ }^{12}$ Hay que recordar que las instituciones educativas no están sujetas a ningún tope.
} 


\section{LA OFENSIVA DE TRUMP CONTRA LAS VISAS DE TRABAJADORES CALIFICADOS}

La llegada de Trump a la presidencia marca un rompimiento tajante con la visión que históricamente habían tenido de la migración los mandatarios de Estados Unidos, como una fuerza positiva para la economía y como parte importante de la herencia cultural del país, aun cuando, como afirman Pierce y Selee (2017), las políticas variaban, tanto en su apertura a los flujos migratorios como en el énfasis en su reforzamiento. Según estos autores, Trump concibe la inmigración como una amenaza mayor para la economía y la seguridad nacional, por lo que ha apoyado cortes dramáticos a la inmigración legal e incluso cuestiona la centralidad de la reunificación familiar (Pierce y Selee, 2017).

Uno de sus objetivos principales es el programa de visas temporales H-1B. Desde su campaña presidencial, Donald Trump anunció reiteradamente el combate que se llevaría a cabo de lo que él pensaba que eran fraudes y abusos por parte de los empresarios que solicitaban estas visas. Decía que se debían tomar pasos mucho más agresivos para limitar su número (Lind, 2017).

Ya en la presidencia, el 18 de abril de 2017 firmó la orden ejecutiva "Compre productos estadunidenses y contrate estadunidenses", con la que buscaba crear más y mejores empleos y lograr remuneraciones más altas para sus connacionales. ${ }^{13} \mathrm{Se}-$ gún él, para conseguirlo se debía instrumentar una administración rigurosa de las leyes de inmigración. En relación con el tema que nos ocupa, señaló que las H-1B son el enemigo prioritario para la política de contratar estadunidenses (Lind, 2017) y ordenó al DHs que, "en coordinación con otras agencias, fomentara políticas que ayudaran a asegurar que las visas $\mathrm{H}-1 \mathrm{~B}$ fueran otorgadas a los beneficiarios más capacitados o mejor pagados" (USCIS, 2017b).

En efecto, se ha documentado que, desde que tomó posesión la administración Trump, el número de peticiones de evidencias a los solicitantes de estas visas aumentó drásticamente, y las tasas de aprobación comenzaron a declinar. Según la periodista Trisha Thadani, del San Francisco Chronicle, en 2017 se incrementaron a más del doble las solicitudes para H-1B rechazadas en noviembre, en comparación con el mismo mes del año anterior (Thadani, 2017).

El premium process que permitía acelerar el procesamiento de solicitudes fue suspendido temporalmente a partir del 3 de abril de 2017 para todos los trámites regulares

\footnotetext{
${ }^{13}$ El 18 de abril de 2017, el presidente Trump firmó la mencionada orden ejecutiva “Compre productos estadunidenses y contrate estadunidenses", con la que se pretende que aumenten los sueldos y las tasas de empleo para los trabajadores nativos para proteger sus intereses económicos con el cumplimiento y la administración rigurosa de las leyes de inmigración.
} 
y para los permisos que se otorgan por haber obtenido grados académicos en el país, e incluso se señaló que podría también aplicarse a las solicitudes que no están sujetas a topes. Esta medida se justificó con el argumento de que se hacía con la finalidad de atender peticiones que habían estado pendientes por largo tiempo y para poder dar prioridad a las adjudicaciones del estatus de extensiones de H-1B que están cerca del día marcado como doscientos cuarenta (USCIS, 2017a).

En noviembre, Trump publicó una lista de prioridades legislativas para el siguiente año, que incluyeron modificaciones mayores a las visas $\mathrm{H}-1 \mathrm{~B}$, aunque no se sabe todavía en qué van a consistir. Sin embargo, los críticos están escépticos sobre el alcance de estas reformas, pues para lograrlas, el primer mandatario requiere de la cooperación del Congreso, que no ha conseguido desde que tomó posesión. Aunque, hay que recordarlo, para gran parte de la ciudadanía interesada este tipo de acciones contra las visas $\mathrm{H}-1 \mathrm{~B}$ constituye una muestra de que se está cumpliendo con lo prometido (Thadani, 2017).

\section{Conclusiones}

El primer aspecto a recalcar es comprender lo que significa la sustitución de las visas de inmigrantes por las que se consideran en Estados Unidos como de "no inmigrantes", con las que se excluye al empleado extranjero de muchos de los beneficios que gozan los nacionales y los inmigrados de ese país. Se contrata al trabajador en los años en los que es más productivo y después se lo expulsa a pesar de sus contribuciones a la economía local. Es por ello que por mucho tiempo estuvieron prohibidas. Las cosas cambiaron a mediados del siglo xx, cuando el origen de la mayoría de los migrantes dejó de ser europeo. Con su utilización se reconocía la demanda de mano de obra en diversas áreas laborales, a pesar de que la necesidad de esos trabajos en muchos casos no era temporal, sino que respondía al rechazo a integrar a su población a ciudadanos extranjeros, principalmente provenientes de América Latina.

Destaca también el cambio en la política migratoria de fines de ese siglo, cuando la revolución científico-tecnológica obligó a las autoridades a ampliar las visas para trabajadores altamente calificados, cuyo número había sido muy reducido. Las grandes empresas en estas especialidades presionaron al gobierno para transformar el sistema migratorio tradicional e incrementar la aceptación de extranjeros por motivos laborales, por encima de aquellos que llegaban por reunificación familiar, dándole prioridad, además, la selección de los más calificados.

Sin embargo, aunque se incrementó la cantidad de inmigrantes por motivos laborales a ciento cuarenta mil anuales, las expectativas de las empresas eran mucho 
mayores, por lo que se recurrió otra vez a las visas de trabajo temporal, creándose un abanico de ellas para que atendieran las necesidades de negocios e instituciones de todo tipo (científicas, artísticas, del espectáculo, los deportes, etc.), pero donde las visas H-1B se convirtieron en las principales. Sus cifras crecieron rápidamente, apoyadas por una legislación muy favorecedora y autoridades poco preocupadas por supervisar que se estuviera cumpliendo con la normatividad correspondiente.

Aunque se usó como gancho el ofrecimiento de la residencia definitiva para cuando los extranjeros terminaran su estancia temporal, en realidad el número de solicitudes rebasa con mucho el tope anual de ciento cuarenta mil, a lo que se agrega el límite del 7 por ciento por país de origen, por lo que muchos de estos portadores de visas $\mathrm{H}-1 \mathrm{~B}$, sobre todo indios y chinos, se mantienen por años en una situación muy incierta antes de conseguir la anhelada green card. Parecía que, a pesar de todo, la tendencia al alza de este tipo de visas se mantendría; sin embargo, el advenimiento del presidente Trump a principios del 2017 cambió el panorama para las grandes empresas científicas y tecnológicas.

Basado en las numerosas quejas de trabajadores desplazados, que atribuyen su situación a la inmigración más que a los cambios tecnológicos, Trump decidió, entre otras políticas migratorias restrictivas, modificar el rumbo de las visas $\mathrm{H}-1 \mathrm{~B}$, a las que culpa de los males que aquejan a los descontentos con el sistema. Durante los casi dos años que ha gobernado hemos atestiguado signos preocupantes en relación con distintos aspectos de la política migratoria. Entre ellos se encuentran los dirigidos a las visas H-1B, a las que inmediatamente comenzó a imponer cambios administrativos que implicaban una mayor supervisión de la forma en que se asignaban, además de que solicitó a las agencias gubernamentales relacionadas que realizaran una revisión de fondo que permita implementar cambios sustanciales.

Ahora bien, independientemente de las medidas que tome el presidente, la revolución científico-tecnológica continúa avanzando y ningún país desarrollado cuenta con la fuerza laboral necesaria para seguir alimentándola únicamente con trabajadores locales. De hecho, ya en 2009, de acuerdo con datos de la Current Population Survey, el 45 por ciento de los científicos médicos y el 37 por ciento de los programadores de cómputo en Estados Unidos eran extranjeros, sobresaliendo también con números muy altos otros científicos y profesionistas, como médicos, ingenieros, especialistas en educación superior, contadores y auditores, enfermeras y arquitectos (Orrenius y Zavodny, 2010: 8-9). Es por ello que las grandes empresas de punta se han dirigido varias veces al presidente para demandarle moderación en su política hacia los inmigrantes altamente calificados, para evitar un retroceso del país, ante un panorama de competencia en el que otras naciones avanzan en el desarrollo del conocimiento. 


\section{FUENTES}

Alarcón, Rafael

2000 "Migrants of the Information Age: Indian and Mexican Engineers and Regional Development in Silicon Valley", Working Paper no. 16, San Diego, Center for Comparative Immigration Studies, Universidad de California, en $<$ https: / ccis. ucsd.edu/_files/wp16.pdf>.

\section{Aquevedo, Eduardo}

2000 "Reestructuración, flexibilidad y trabajo en América Latina: un nuevo 'orden' económico internacional", Papeles de Población, vol. 6, no. 26, octubre-diciembre, pp. 89-107, en <http:/ / www.scielo.org.mx/ scielo.php?script=sci_arttex t\&pid=S1405-74252000000400005>.

\section{Corona, LeONel}

1991 "Revolución científico-técnica", en Leonel Corona, coord., México ante las nuevas tecnologías, México, Centro de Investigaciones Interdisciplinarias en Ciencias y Humanidades (CEIICH), UNAM, pp. 17-37.

HIRA, RON

2010 "Bridge to Immigration or Cheap Temporary Labor? H-1B \& L-1 Visas Are a Source of Both", Briefing Paper no. 257, Economic Policy Institute (EPI), en $<$ http://s4.epi.org/files/page/-/pdf/bp257.pdf>.

Jachimowicz, Maia y Deborah W. Meyers

2002 “Temporary High-skilled Migration”, Migration Policy Institute, en <https:// www.migrationpolicy.org/article/temporary-high-skilled-migration/\#top >.

LIND, DARA

2017 "What Trump's H-1B Visa Executive Order Actually Does", Vox, 19 de abril, en <http://www.vox.com/policy-and-politics / 2017/4/18/15340686/h1b-visaexecutive-order-trump $>$.

MARTin, PhILIP L.

2003 Highly Skilled Labor Migration: Sharing the Benefits, Ginebra, International Institute for Labour Studies, International Labour Organization (ILO). 
MehtA, CYrus D.

2004 "US Immigration Laws: Emerging Trends in Policies and Procedures", ILW. com (Immigration Law Publisher), en <http: / / www.ilw.com/articles / 2004,0325-mehta.pdf>.

Meyers, Deborah Waller

2006 "Temporary Worker Programs: A Patchwork Policy Response", Task Force Insight no. 12 (enero), Migration Policy Institute, Washington, D.C., en <https:/ / www.migrationpolicy.org / research/temporary-worker-programs-pat chwork-policy-response>.

Office of Foreign Labor Certification (OFlc)

2016 "Annual Report", Employment and Training Administration, U.S. Department of Labor, en <https: / /www.foreignlaborcert.doleta.gov/pdf/OFLC_Annual_ Report_FY2016.pdf $>$.

Orrenius, Pia y Madeline Zavodny

2010 "From Brawn to Brains: How Immigration Works for America, 2010 Annual Report", Federal Reserve Bank of Dallas, en <https: / www.dallasfed.org/ / media/ documents / fed/annual/2010/ar10b.pdf $>$.

Pierce, Sarah y Andrew Selee

2017 "Immigration under Trump: A Review of Policy Shifts in the Year since the Election", diciembre, Migration Policy Institute, en <https://www.migra tionpolicy.org/research/immigration-under-trump-review-policy-shifts $>$.

Preston, Julia

2015 "Miscalculation on Visas Disrupts Lives of Highly Skilled Immigrants", The New York Times, 15 de octubre, en <http: / / www.nytimes.com/2015/10/02/ us / incorrect-count-on-visas-disrupts-lives-of-highly-skilled-immigrants. html?_r=1>.

Ruiz, Neil G., Jill H. Wilson y Shyamali Choudhury

2012 "The Search for Skills: Demand for H-1B Immigrant Workers in U.S. Metropolitan Areas", Brookings, 18 de julio, en <https: / www.brookings.edu/ research/the-search-for-skills-demand-for-h-1b-immigrant-workers-in-u-smetropolitan-areas/>. 
THADANI, TRISHA

2017 "More H-1B Hopefuls Denied under Trump, Data Show", San Francisco Chronicle, 17 de diciembre, en <https:// www.sfchronicle.com/business/article / More-H-1B-hopefuls-denied-under-Trump-data-show-12437578.php>.

Trigueros, Paz

2015 "La contratación de trabajadores agrícolas con visas H-2A. Del Programa Bracero a la situación actual", en Martha Judith Sánchez Gómez y Sara María Lara Flores, coords., Los programas de trabajadores agrícolas temporales. ¿Una solución a los retos de las migraciones en la globalización?, México, Instituto de Investigaciones Sociales (IIS), UNAM, pp. 173-206.

2012a "La migración calificada en Estados Unidos. El caso de las visas temporales", en Ana María Aragonés, coord., Migración internacional. Algunos desafíos, México, Instituto de Investigaciones Económicas (IIEC), UNAM, pp. 75-100.

2012b “Trabajadores migrantes temporales altamente calificados en Estados Unidos, política migratoria, visas y características actuales", en Roberto Benencia, Fernando Herrera y Elaine Levine, coords., Ser migrante latinoamericano, ser vulnerable, trabajar precariamente, Madrid, Anthropos/Universidad Autónoma Metropolitana, pp. 155-171.

\section{U. S. Citizenship and Immigration Services (uscis)}

2017a "USCIS Will Temporarily Suspend Premium Processing for All H-1B Petitions", 3 de abril, en <https: / www.uscis.gov/news/alerts/uscis-will-temporarily-sus pend-premium-processing-all-h-1b-petitions $>$.

2017b “¿Cómo se utiliza el Servicio de Procesamiento Prioritario?”, 28 de agosto, en $<$ https: / / www.uscis.gov / es / formularios / como-se-utiliza-el-servicio-deprocesamiento-prioritario $>$.

2017c “Compre productos estadounidenses y contrate estadounidenses: colocando a los trabajadores estadounidenses primero", 24 de octubre, en <https:// www.uscis.gov / es / recursos / compre-productos-estadounidenses-y-contra te-estadounidenses-colocando-los-trabajadores-estadounidenses-primero>.

2006 "Temporary Migration to the United States: Nonimmigrant Admissions un der U.S. Immigration Law", enero, U.S. Immigration Report Series, en <https: / / www.sru.edu/Documents / offices / payroll/ uscis-temp-migr-to-us.pdf>.

U.S. Department of Homeland Security (DHS)

2018 "Yearbook of Immigration Statistics", en <https:/ / www.dhs.gov/immigra tion-statistics / yearbook>. 
2017a "Yearbook of Immigration Statistics 2016", en <https:/ / www.dhs.gov/im migration-statistics/yearbook/2016>.

$2017 b$ "Characteristics of H-1B Specialty Occupation Workers. Fiscal Year Annual Report to Congress, October 1, 2015-September 30, 2016", en <https: / / www. uscis.gov / sites / default / files / uscis / Resources / Reports\%20and\%20Studies / H-1B/H-1B-FY16.pdf>.

2003 "Yearbook of Immigration Statistics", en < https:/ / www.dhs.gov/immigra tion-statistics/yearbook/2003>.

U.S. DEPARTMENT OF JUSTICE (USDOJ)

1986 Immigration and Naturalization Service Annual Report, Washington, D. C. [1978].

1980 "Table 16B", 1978 Statistical Yearbook of the Immigration and Naturalization Service, pp. 44-48, en <https: / / archive.org/ details / statisticalyearb1978unit>.

WASEM, Ruth ELLEN

2006 "Immigration Policy for Intracompany Transfers (L Visa): Issues and Legislation", Congressional Research Service, en <https: / www.researchgate.net/ publication/324262384_Immigration_Policy_for_Intracompany_ Transfers_L_Visa_Issues_and_Legislation $>$.

Wasem, Ruth Ellen y Geoffrey K. Collver

2001 "Immigration of Agricultural Guest Workers: Policy, Trends, and Legislative Issues", Congressional Research Service, en <https: / / digitalcommons.ilr. cornell.edu / cgi / viewcontent.cgi?referer=https: / / www.google.com.mx / \& httpsredir=1\&article=1031\&context=key_workplace $>$.

Wong, Margaret

2010 The Immigrant's Way: For All Immigrants, by an Immigrant, 3a. ed., Nueva York, en $<$ ILW.com>. 